\title{
EXISTENCE THEOREMS FOR DOUBLE INTEGRAL PROBLEMS OF THE CALCULUS OF VARIATIONS. $\dagger$
}

\author{
BY \\ E. J. MCSHANE
}

For single-integral problems of the calculus of variations there are in the literature a number of existence theorems of considerable generality. Recently Tonelli has established several existence theorems for double integral problems of the form $\iint f\left(x, y, z, z_{x}, z_{y}\right) d x d y=\min$. But to the best of my knowledge, except for the several discussions of the problem of Plateau the literature contains no proof of any existence theorem for double-integral problems in parametric form, that is, for problems of the form $F(S)$ $=\iint f(x, y, z, X, Y, Z) d u d v=\min$, where the equations $x=x(u, v), y=y(u, v)$, $z=z(u, v)$ represent a surface and $X, Y, Z$ are the three jacobians of $(x, y, z)$ with respect to $(u, v)$.

The present paper gives the proof of two such theorems, in each of which the integrand function is permitted to be a function of $(X, Y, Z)$ of quite general type, but is required to be independent of the coordinates $(x, y, z)$. The theorems are based on a semi-continuity proof and a convergence theorem. The semi-continuity of quasi-regular functionals $F(S)$ I have already established under conditions of adequate generality. Here I develop the convergence theorem needed. The methods are extensions of those previously used in connection with the problem of Plateau.

1. Preliminary remarks. The word surface will always be used to mean a continuous surface of the type of the circle, represented by three equations $x=x(u, v), y=y(u, v), z=z(u, v)$, where $(u, v)$ ranges over the interior and boundary of a Jordan region $B$ (i.e., a region bounded by a simple closed curve). In case the six partial derivatives $x_{u}, x_{v}$, etc., all exist and are finite, we denote the three jacobians of $x, y, z$ with respect to $u, v$ by the symbols $X, Y, Z$ :

$$
X=\left|\begin{array}{ll}
y_{u} & z_{u} \\
y_{v} & z_{v}
\end{array}\right|, \quad Y=\left|\begin{array}{cc}
z_{u} & x_{u} \\
z_{v} & x_{v}
\end{array}\right|, \quad Z=\left|\begin{array}{ll}
x_{u} & y_{u} \\
x_{v} & y_{v}
\end{array}\right| .
$$

Let us suppose that $f(X, Y, Z)$ is a function positively homogeneous of degree 1 in $(X, Y, Z)$ and continuous together with its first partial derivatives for all $(X, Y, Z) \neq(0,0,0)$. For all arguments $(X, Y, Z)$ such that $X^{2}+Y^{2}+Z^{2}$

$\dagger$ Presented to the Society, October 28, 1933; received by the editors July 28, 1934.

$\ddagger$ E. J. McShane, Parametrizations of saddle surfaces, etc., these Transactions, vol. 35 (1933), pp. 716-733. This paper will henceforth be cited as S.S. 
$=1$ the function $f$ is bounded, say $|f| \leqq M$; hence by homogeneity the inequality

$$
|f(X, Y, Z)| \leqq M\left[X^{2}+Y^{2}+Z^{2}\right]^{1 / 2} \leqq M(|X|+|Y|+|Z|)
$$

holds for all $X, Y, Z$. Consequently, if $x=x(u, v), y=y(u, v), z=z(u, v),(u, v)$ on $B$, is a representation of a surface such that the six partial derivatives $x_{u}$, etc., are defined almost everywhere in $B$, and the jacobians $X, Y, Z$ are summable over the set on which they are defined, it follows that the integral

$$
\iint_{B} f(X, Y, Z) d u d v
$$

exists. $\dagger$

As in the case of single integrals, the mere existence of this integral is inadequate for our purposes. $\ddagger$ In the study of the parametric problem (single integrals) we restrict ourselves to representations $x=x(t)$, etc., in which the functions $x(t), \cdots$ are absolutely continuous. Lacking an adequate generalization of the notion of absolute continuity to the pairs of functions of two variables, we say that a surface $S$ with finite Lebesgue area $L(S)$ is an admissible surface (for the integrand $f(X, Y, Z)$ ) if $S$ has representation $x=x(u, v), y=y(u, v), z=z(u, v),(u, v)$ on $B$, for which the jacobians $X, Y, Z$ are defined almost everywhere in $B$ and for which the following approximation property holds: there exists a sequence $\left\{\pi_{n}\right\}$ of polyhedra $\pi_{n}: x=x_{n}(u, v)$, $y=y_{n}(u, v), z=z_{n}(u, v),(u, v)$ on $B_{n}$, such that $\lim \pi_{n}=S, \lim L\left(\pi_{n}\right)<\infty$, and $\lim \iint_{B_{n}} f\left(X_{n}, Y_{n}, Z_{n}\right) d u d v=\iint_{B} f(X, Y, Z) d u d v$. The representation $x=x(u, v)$, etc., is then called an admissible representation of the surface $S$.

If for the corresponding single-integral problem we write the analogous definition of admissible curves, we find that for every integral $\int f\left(x, y, z, x^{\prime}, y^{\prime}, z^{\prime}\right) d t$ the class of admissible curves is the same as the class of rectifiable curves; and if in addition the integral is positive definite (i.e., $f>0$ whenever $\left.\left(x^{\prime}, y^{\prime}, z^{\prime}\right) \neq(0,0,0)\right)$ and positive quasi-regular, the admissible representations are the same as the absolutely continuous representations. For double integrals no such simple characterization is at present known. But it can be stated that for every integrand $f(X, Y, Z)$ with the continuity and homogeneity properties above described the class of admissible surfaces

$\dagger$ The integrand may be undefined on a set of measure 0 . Here and henceforth we agree that if a function $\phi(u)$ is defined at all points of a set $E$ except those of a set $N$ of measure 0 and is summable on $E-N$, the symbol $\int_{E} \phi(u) d u$ shall mean the integral $\int_{E-N} \phi(u) d u$.

$\ddagger$ As has been shown by M. Lavrentieff, Sur quelques problèmes du calcul des variations, Annali di Matematica, (4), vol. 4 (1927), p. 7. 
includes the class of surfaces of "type $\dagger C$," which in turn includes the class of all continuous surfaces having representations $x=x(u, v), y=y(u, v)$, $z=z(u, v),(u, v)$ on $B$, which satisfy the conditions

(1.1a) for almost all numbers $K$, the functions $x(u, v), y(u, v), z(u, v)$ are absolutely continuous functions of $u$ on every segment of the line $v=K$ lying interior to $B$, and are absolutely continuous functions of $v$ on every segment of the line $u=K$ lying interior to $B$;

(1.1b) the six partial derivatives $x_{u}, x_{v}, y_{u}, y_{v}, z_{u}, z_{v}$ (which by (1.1a) exist almost everywhere in $B$ ) are summable together with their squares over the region $B$.

For surfaces of type $C$ typically represented (in particular, for representations satisfying conditions (1.1)) we already know $\ddagger$ that the value of the integral is independent of the particular representation and is thus a functional of the surface alone. We then have the right to denote the integral by the symbol $F(S)$,

$$
F(S)=\iint f(X, Y, Z) d u d v
$$

But for admissible surfaces not of type $C$ it is not known that this invariance property holds; hence for general admissible surfaces we shall always write the integral in full, avoiding the (possibly multiple-valued) symbol $F(S)$.

We define the Weierstrass $\varepsilon_{\text {-function as usual: }}$

$$
\begin{aligned}
\varepsilon(X, Y, Z, \bar{X}, \bar{Y}, \bar{Z})= & f(\bar{X}, \bar{Y}, \bar{Z})-\bar{X} f_{X}(X, Y, Z) \\
& -\bar{Y} f_{Y}(X, Y, Z)-\bar{Z} f_{Z}(X, Y, Z) ;
\end{aligned}
$$

and as usual we call $F(S)$ positive quasi-regular if $\mathcal{E}(X, Y, Z, \bar{X}, \bar{Y}, \bar{Z}) \geqq 0$ for all $(\bar{X}, \bar{Y}, \bar{Z})$ and all $(X, Y, Z) \neq(0,0,0)$, and we call it positive definite if $f(X, Y, Z)>0$ for all $(X, Y, Z) \neq(0,0,0)$.

We shall say that a surface $S$ is of type $L_{2}$ if it possesses a representation

$$
x=x(u, v), y=y(u, v), z=z(u, v), u^{2}+v^{2} \leqq 1,
$$

in which the functions $x(u, v)$, etc., satisfy conditions (1); the representation (1.4) we shall correspondingly call a typical representation. It is known§ that if a surface $S$ has a representation

$\dagger$ Defined and studied in Integrals over surfaces in parametric form, Annals of Mathematics, vol. 34 (1933), p. 815; cf. also C. B. Morrey, A class of representations of manifolds, American Journal of Mathematics, vol. 55 (1933), p. 701.

$\ddagger$ E. J. McShane, loc. cit. in the preceding footnote.

$\S$ E. J. McShane, On the minimizing property of the harmonic function, Bulletin of the American Mathematical Society, vol. 40 (1934), p. 593. 


$$
x=x(u, v), y=y(u, v), z=z(u, v),(u, v) \text { on } B,
$$

which satisfies conditions (1.1), it is necessarily of type $L_{2}$; but we shall not make use of this.

2. A transformation of the integrand. By change of coordinates we can bring the integrands under consideration into a special form, useful for later proofs.

LEMMA 2.1. Let the inequality

$$
f(X, Y, Z)+f(-X,-Y,-Z)>0
$$

hold for all $(X, Y, Z) \neq(0,0,0)$. There exists a linear transformation

$$
\begin{aligned}
& X=A_{1} X^{\prime}+B_{1} Y^{\prime}+C_{1} Z^{\prime}, \\
& Y=A_{2} X^{\prime}+B_{2} Y^{\prime}+C_{2} Z^{\prime}, \\
& Z=A_{3} X^{\prime}+B_{3} Y^{\prime}+C_{3} Z^{\prime},
\end{aligned}
$$

of determinant 1 , such that the function

$$
\phi\left(X^{\prime}, Y^{\prime}, Z^{\prime}\right)=f(X, Y, Z)
$$

satisfies the conditions

$$
\begin{aligned}
& \phi_{Y^{\prime}}(1,0,0)=\phi_{Y}(-1,0,0) \\
& \phi_{Z}(1,0,0)=\phi_{Z}(-1,0,0) \\
& \phi_{Z}(0,1,0)=\phi_{Z}(0,-1,0) \\
& \phi_{X^{\prime}}(0,1,0)=\phi_{X}(0,-1,0) .
\end{aligned}
$$

Let us define

$$
g(X, Y, Z) \equiv f(X, Y, Z)+f(-X,-Y,-Z) .
$$

The function $g$ obviously has the same differentiability and homogeneity properties as $f$, and the surface $S$ in $X Y Z$-space defined by the equation

$$
g(X, Y, Z)=1
$$

is symmetrical with respect to the origin. (By (2.1) the surface exists, and in the direction of the unit vector $X_{u}, Y_{u}, Z_{u}$ has the distance from the origin $r=\left[g\left(X_{u}, Y_{u}, Z_{u}\right)\right]^{-1}$.) Moreover, from the homogeneity relation

$$
X g_{X}+Y g_{Y}+Z g_{Z}=g=1
$$

we see that the three derivatives cannot vanish simultaneously, so that the surface $S$ is continuously differentiable.

On $S$ there is a point at maximum distance from the origin. By a rotation of axes we bring this point to the $X$-axis. Then for $Y=Z=0$ the tangent plane to $S$ is parallel to the $Y Z$-plane. We now introduce polar coordinates, 
$r, \theta, \phi$ so that $X=r \sin \phi, Y=r \cos \phi \cos \theta, Z=r \cos \phi \sin \theta$. The surface $S$ can then be represented in the form

$$
r=r(\theta, \phi),
$$

where $r(\pi+\theta,-\phi)=r(\theta, \phi)$. Since this implies

$$
\frac{\partial}{\partial \phi} r(\pi+\theta, 0)=-\frac{\partial}{\partial \phi} r(\theta, 0)
$$

and this derivative is continuous, there exists a point, with arguments $\left(\theta_{0}, 0\right)$, at which $\partial r / \partial \phi$ vanishes. By rotation about the $X$-axis we bring this point to the $Y$-axis, so that $\partial r / \partial \phi$ vanishes for $\theta=\phi=0$ (that is, for $X=Z=0$ ). The tangent plane at $\theta=\phi=0$ is then parallel to the $X$-axis, but not necessarily to the $Z$-axis. Let $l_{1}$ be the line through the origin parallel to the intersection of that tangent plane with the $Y Z$-plane. By an affine transformation $T$ of the form $X=\bar{X}, Y=\bar{Y}+K \bar{Z}, Z=\bar{Z}$, we bring $l_{1}$ to the $Z$-axis, leaving the $X$ and $Y$ axes unchanged. After this transformation the tangent plane at $X=Z=0$ is parallel to the $X Z$-plane; the tangent plane at $Y=Z=0$ and the $Y Z$-plane are unchanged, hence remain parallel.

The two rotations and the affine transformation $T$ can be combined into a single linear transformation of the form (2.2). In terms of the new coordinates, the surface $S$ has the equation

$$
\phi\left(X^{\prime}, Y^{\prime}, Z^{\prime}\right)+\phi\left(-X^{\prime},-Y^{\prime},-Z^{\prime}\right)=1
$$

where $\phi$ is defined by equation (2.3). The normal to $S$ has the direction numbers (dropping primes)

$$
\begin{aligned}
& \phi_{X}(X, Y, Z)-\phi_{X}(-X,-Y,-Z), \\
& \phi_{Y}(X, Y, Z)-\phi_{Y}(-X,-Y,-Z), \\
& \phi_{Z}(X, Y, Z)-\phi_{Z}(-X,-Y,-Z) .
\end{aligned}
$$

But for $Y=Z=0$ the normal has direction cosines $( \pm 1,0,0)$, so that the last two of the numbers (2.7) are 0 for $Y=Z=0$ whether the positive or negative value of $X$ be chosen. Recalling that $\phi_{Y}$ and $\phi_{Z}$ are positively homogeneous of degree 0 , this yields the first pair of equations (2.4). For $X=Z=0$ the normal has direction cosines $(0, \pm 1,0)$; this likewise yields the second pair of equations (2.4).

Let us suppose that we are given an integrand $f(X, Y, Z)$ satisfying inequality (2.1), and let the matrix

$$
\left(\begin{array}{lll}
a_{1} & a_{2} & a_{3} \\
b_{1} & b_{2} & b_{3} \\
c_{1} & c_{2} & c_{3}
\end{array}\right)
$$


be the reciprocal of the matrix of the transformation (2.2). We find readily that for every surface

$S:$

$$
x=x(u, v), y=y(u, v), z=z(u, v),(u, v) \text { on } B,
$$

for which $f(X, Y, Z)$ is summable the transformation

$$
\begin{aligned}
& x=a_{1} x^{\prime}+b_{1} y^{\prime}+c_{1} z^{\prime}, \\
& y=a_{2} x^{\prime}+b_{2} y^{\prime}+c_{2} z^{\prime}, \\
& z=a_{3} x^{\prime}+b_{3} y^{\prime}+c_{3} z^{\prime}
\end{aligned}
$$

induces transformation (2.7) on the jacobians $X, Y, Z$, and

$$
\iint_{B} \phi\left(X^{\prime}, Y^{\prime}, Z^{\prime}\right) d u d v=\iint_{B} f(X, Y, Z) d u d v .
$$

The surfaces admissible for $f$ transform into surfaces admissible for $\phi$; likewise the class of surfaces of type $C$ transforms into itself and the class of surfaces of type $L_{2}$ also transforms into itself. Hence if we are given a Jordan curve $\Gamma$, transformed by (2.8) into a curve $\Gamma^{\prime}$ of $x^{\prime} y^{\prime} z^{\prime}$-space, the problem of finding a minimizing surface for $\iint f(X, Y, Z) d u d v$ in the class of all surfaces bounded by $\Gamma$ and belonging to any one of the three analytic classes just mentioned is equivalent to the problem of finding a minimizing surface for $\iint \phi\left(X^{\prime}, Y^{\prime}, Z^{\prime}\right) d u d v$ in the class of all surfaces bounded by $\Gamma^{\prime}$ and belonging to the corresponding analytic class. In other words, there is no loss of generality in assuming to begin with that $f(X, Y, Z)$ satisfies the equations

$$
\begin{aligned}
& f_{Y}(1,0,0)=f_{Y}(-1,0,0), \\
& f_{Z}(1,0,0)=f_{Z}(-1,0,0), \\
& f_{Z}(0,1,0)=f_{Z}(0,-1,0), \\
& f_{X}(0,1,0)=f_{X}(0,-1,0) .
\end{aligned}
$$

3. First existence theorem for positive definite integrals. In this section we shall consider integrands $f(X, Y, Z)$ which satisfy the condition

$$
f(X, Y, Z)>0 \text { for }(X, Y, Z) \neq(0,0,0) \text {. }
$$

Given a Jordan curve $\Gamma$, it is clear that the greatest lower bound $i$ of $\iint f(X, Y, Z) d u d v$ for all admissible surfaces bounded by $\Gamma$ is non-negative. Another lower bound associated with $\Gamma$ we define in the following way:

Let $S_{n}: x=x_{n}(u, v)$, etc., $n=1,2, \cdots$, be a sequence of admissible surfaces whose boundaries tend to $\Gamma$, and let $m\left(\left\{S_{n}\right\}\right)$ be the lower limit of $\iint f\left(X_{n}, Y_{n}, Z_{n}\right) d u d v$. We define $m$ to be the greatest lower bound of the numbers $m\left(\left\{S_{n}\right\}\right)$ for all such sequences $\left\{S_{n}\right\}$. Clearly 


$$
m \leqq i,
$$

for we can construct a sequence $\left\{S_{n}\right\}$ of admissible surfaces bounded by $\Gamma$ for which the integrals tend to $i$, and $m$ is not greater than the limit of the integrals over the surfaces $S_{n}$.

We now proceed to the proof of

THEOREM 3.1. Let the integral

$$
\iint f(X, Y, Z) d u d v
$$

be positive definite and positive quasi-regular, and let the curve

$\Gamma:$

$$
x=x(t), y=y(t), z=z(t)
$$

be a Jordan curve in xyz-space, bounding at least one admissible surface. $\dagger$ Then there exists a triple of functions $x(u, v), y(u, v), z(u, v)$, defined for $u^{2}+v^{2} \leqq 1$, with the following properties:

(1) the surface

$$
S: \quad x=x(u, v), y=y(u, v), z=z(u, v), u^{2}+v^{2} \leqq 1,
$$

is bounded by the curve $\Gamma$; that is, the equations $x=x(\cos \theta, \sin \theta), y=y(\cos \theta$, $\sin \theta), z=z(\cos \theta, \sin \theta), 0 \leqq \theta \leqq 2 \pi$, form a representation $\ddagger$ of the curve $\Gamma$;

(2) the functions $x(u, v), y(u, v), z(u, v)$ satisfy conditions (1.1);

(3) the surface (3.4) minimizes the integral (3.3) in the class of all admissible surfaces bounded by $\Gamma$, and in fact

$$
F(S)=i=m \text {. }
$$

In accordance with the remark at the end of $\$ 2$, there is no loss of generality in assuming that equations (2.10) are satisfied. By the homogeneity of $f$ we have

$$
f_{Z}(0,0,1)=f(0,0,1), f_{Z}(0,0,-1)=-f(0,0,-1) .
$$

By hypothesis inequality (3.1) is valid; and from (3.6) and (3.1) we see that there exist numbers $a, b$ such that

$$
\begin{aligned}
& a f_{Z}(0,0,1)+f_{X}(0,0,1)=a f_{Z}(0,0,-1)+f_{X}(0,0,-1), \\
& b f_{Z}(0,0,1)+f_{Y}(0,0,1)=b f_{Z}(0,0,-1)+f_{Y}(0,0,-1) .
\end{aligned}
$$

† From the results of S.S. (Lemma 3 and Theorem $I$ ) this is equivalent to requiring that $\Gamma$ bound at least one surface of finite area.

$\ddagger$ But in this representation it is possible that two distinct points $\left(\cos \theta_{1}, \sin \theta_{1}\right)$ and $\left(\cos \theta_{2}\right.$, $\left.\sin \theta_{2}\right)$ might yield the same point $(x, y, z)$. 
We define the number $k$ by the relation

$$
k=2\left(1+a^{2}+b^{2}\right) .
$$

Let us now select a sequence of admissible surfaces

$S_{n}: \quad x=x_{n}^{*}(u, v), y=y_{n}^{*}(u, v), z=z_{n}^{*}(u, v),(u, v)$ on $B_{n}$,

bounded by curves $\Gamma_{n}^{*}$ such that $\Gamma_{n}^{*} \rightarrow \Gamma$ and such that

$$
\lim _{n \rightarrow \infty} \iint_{B_{n}} f\left(X_{n}^{*}, Y_{n}^{*}, Z_{n}^{*}\right) d u d v=m .
$$

Since the $S_{n}$ are admissible surfaces, we can for each $S_{n}$ find a polyhedron $\pi_{n}$, which we assume to have non-degenerate triangles for faces, such that

$$
\operatorname{dist}\left(\pi_{n}, S_{n}\right)<1 / 2^{n}
$$

and

$$
\left|F\left(\pi_{n}\right)-\iint_{B_{n}} f\left(X_{n}^{*}, Y_{n}^{*}, Z_{n}^{*}\right) d u d v\right|<\frac{1}{2^{n}} .
$$

From (3.10) and the relation $\Gamma_{n}^{*} \rightarrow \Gamma$ we see that the boundary curves $\Gamma_{n}$ of the polyhedra $\pi_{n}$ satisfy

$$
\Gamma_{n} \rightarrow \Gamma
$$

and from (3.11) and (3.9) we see that

$$
F\left(\pi_{n}\right) \rightarrow m \text {. }
$$

It is known $\dagger$ that every polyhedron $\pi$ with non-degenerate faces admits of a parametric representation of the following kind.

(a) The functions representing $\pi$ are defined in the unit circle; that is, $\pi$ is represented by equations

$$
x=x(u, v), y=y(u, v), z=z(u, v), u^{2}+v^{2} \leqq 1 .
$$

(b) The unit circle is subdivided by arcs into a finite number of curvilinear triangles $\delta_{1}, \cdots, \delta_{k}$ and equations (3.14) carry each triangle into a rectilinear triangle in $x y z$-space.

(c) The triangles $\delta_{i}$ are bounded by arcs which are analytic, including end points.

(d) Interior to each triangle $\delta_{i}$ the functions $x(u, v), y(u, v), z(u, v)$ are analytic and satisfy the relations

† See, e.g., Caratheodory, Conformal Representation (No. 28 of the Cambridge Tracts in Mathematics and Physics), chapter VII. 


$$
E=G, \quad F=0 .
$$

(e) Three arbitrarily given distinct points $A_{1}, A_{2}, A_{3}$ on the boundary curve of $\pi$ correspond to three arbitrarily given distinct points $A_{1}^{*}, A_{2}^{*}, A_{3}^{*}$ on the unit circle $u^{2}+v^{2}=1$.

Accordingly, we choose on the curve $\Gamma$ three distinct points $A_{1}, A_{2}, A_{3}$, and on each $\Gamma_{n}$ we choose three distinct points $A_{1}{ }^{(n)}, A_{2}{ }^{(n)}, A_{3}{ }^{(n)}$ such that

$$
\lim _{n \rightarrow \infty} A_{i}{ }^{(n)}=A_{i} \quad(i=1,2,3) .
$$

On the circumference of the unit circle $u^{2}+v^{2} \leqq 1$ (which circle we shall henceforth denote by $K$ ) we choose three distinct points $A_{1}^{*}, A_{2}^{*}, A_{3}^{*}$, and we represent each polyhedron $\pi_{n}$ by equations

$$
\pi_{n}: \quad x=x_{n}(u, v), y=y_{n}(u, v), z=z_{n}(u, v), u^{2}+v^{2} \leqq 1,
$$

such that conditions (a), (b), (c), (d) are satisfied and the points $A_{1}{ }^{(n)}, A_{2}{ }^{(n)}$, $A_{3}{ }^{(n)}$ correspond to $A_{1}^{*}, A_{2}^{*}, A_{3}^{*}$ respectively.

Let $\mu>0$ be the lower bound of $f(X, Y, Z)$ on the bounded closed set $X^{2}+Y^{2}+Z^{2}=1$; then for all $X, Y, Z$ we have

$$
f(X, Y, Z) \geqq \mu\left[X^{2}+Y^{2}+Z^{2}\right]^{1 / 2} .
$$

We may assume without loss of generality that $F\left(\pi_{n}\right)<m+1$ for all $n$; whence

$$
\begin{aligned}
m+1 & >\iint_{K} f\left(X_{n}, Y_{n}, Z_{n}\right) d u d v \geqq \iint_{K} \mu\left[X_{n}^{2}+Y_{n}^{2}+Z_{n}^{2}\right]^{1 / 2} d u d v \\
& =\mu \iint_{K}\left[E_{n} G_{n}-F_{n}^{2}\right]^{1 / 2} d u d v=\frac{\mu}{2} \iint_{K}\left(E_{n}+G_{n}\right) d u d v,
\end{aligned}
$$

so that

$$
\iint_{K}\left(E_{n}+G_{n}\right) d u d v \leqq H, \quad H=2(m+1) / \mu .
$$

On the functions (3.16) we now operate to reduce their monotonic deficiency. $\nmid$ We choose a cube $d \leqq x \leqq d+h, d \leqq y \leqq d+h, d \leqq z \leqq d+h$ large enough to include the whole curve $\Gamma$ and all the curves $\Gamma_{n}$ in its interior (as is possible, since $\left.\Gamma_{n} \rightarrow \Gamma\right)$. The set of points $(u, v)$ such that $z_{n}(u, v)>d$ is an open set, except that it may contain limit points on the circumference of the unit circle $K$, and it consists of a finite number of maximal connected subsets. We reject those subsets which have points in common with the circumference of $K$, and name the rest $R_{1}, \cdots, R_{p}$. We proceed similarly with the set $z_{n}(u, v)<d$;

† S.S., p. 717. 
the maximal connected portions of this set which have no point in common with the circumference of the unit circle we call $R_{p+1}, R_{p+2}, \cdots, R_{q}$.

On each $R_{i}$ we define the functions $\xi^{(1)}(u, v), \eta^{(1)}(u, v), \zeta^{(1)}(u, v)$ by the relations

$$
\xi^{(1)}=x_{n}+a\left(z_{n}-d\right), \eta^{(1)}=y_{n}+b\left(z_{n}-d\right), \zeta^{(1)}=d ;
$$

on the remainder $K-\sum R_{i}$ of the unit circle we set

$$
\xi^{(1)} \equiv x_{n}, \eta^{(1)} \equiv y_{n}, \zeta^{(1)} \equiv z_{n} \text {. }
$$

The functions $\xi^{(1)}, \eta^{(1)}, \xi^{(1)}$ clearly retain properties (a), (b), (c), (e), and the surface

$$
\Sigma^{(1)}: \quad x=\xi^{(1)}(u, v), y=\eta^{(1)}(u, v), z=\zeta^{(1)}(u, v)
$$

is bounded by $\Gamma_{n}$. Moreover, if we denote the jacobians of $\xi^{(1)}, \eta^{(1)}, \zeta^{(1)}$ by $\Xi^{(1)}, H^{(1)}, Z^{(1)}$, we find

$$
\Xi^{(1)}=0, \mathrm{H}^{(1)}=0, \mathrm{Z}^{(1)}=Z_{n}-a X_{n}-b Y_{n},(u, v) \text { on } \sum R_{i},
$$

so that

$$
\begin{aligned}
\iint_{K}\left[f\left(X_{n}, Y_{n}, Z_{n}\right)-f\left(\Xi^{(1)}, \mathrm{H}^{(1)}, \mathrm{Z}^{(1)}\right)\right] d u d v \\
=\iint_{\Sigma R_{i}}\left[\mathcal{E}\left(0,0, \mathrm{Z}^{(1)}, X_{n}, Y_{n}, Z_{n}\right)-Y_{n} f_{Y}\left(0,0, \mathrm{Z}^{(1)}\right)\right. \\
\left.\quad-\left(a X_{n}+b Y_{n}\right) f_{Z}\left(0,0, \mathrm{Z}^{(1)}\right)\right] d u d v \\
\geqq-\sum \iint_{R_{i}}\left\{X_{n}\left[f_{X}\left(0,0, \mathrm{Z}^{(1)}\right)+a f_{Z}\left(0,0, \mathrm{Z}^{(1)}\right)\right]\right. \\
\left.\quad+Y_{n}\left[f_{Y}\left(0,0, \mathrm{Z}^{(1)}\right)+b f_{Z}\left(0,0, \mathrm{Z}^{(1)}\right)\right]\right\} d u d v .
\end{aligned}
$$

Since the derivatives $f_{X}$, etc., are positively homogeneous of degree 0 in $(X, Y, Z)$, it follows from (3.7) that for all $\mathrm{Z}^{(1)} \neq 0$ the equations

$$
\begin{aligned}
& f_{X}\left(0,0, Z^{(1)}\right)+a f_{Z}\left(0,0, Z^{(1)}\right)=f_{X}(0,0,1)=a f_{Z}(0,0,1)=c_{1}, \\
& f_{Y}\left(0,0, Z^{(1)}\right)+b f_{Z}\left(0,0, Z^{(1)}\right)=f_{X}(0,0,1)+a f_{Z}(0,0,1)=c_{2}
\end{aligned}
$$

hold. If $Z^{(1)}=0$ we assign $f_{X}$ the value $f_{X}(0,0,1)$, and likewise for $f_{Y}$ and $f_{Z}$; (3.23) continues to hold, and also the equations (3.24). But for each of the regions $R_{i}$ we have

$$
\iint_{R_{i}} X_{n} d u d v=\int y_{n} z_{n}^{\prime} d s
$$


the single integral being taken around the boundary of $R_{i}$. On the boundary of $R_{i}$ we have $z_{n}=d$, by the definition of $R_{i}$; hence $z_{n}^{\prime}=0$, and

$$
\iint_{R_{i}} X_{n} d u d v=0
$$

From this and (3.23) follows

$$
F\left(\Sigma^{(1)}\right) \leqq F\left(\pi_{n}\right) .
$$

To the surface $\Sigma^{(1)}$, in its representation (3.21), we apply a similar process, the number $d$ being replaced by $d+h / n$ in defining the sets $R_{i}$ and in equations (3.19). We thus obtain a surface

$$
\Sigma^{(2)}: \quad x=\xi^{(2)}(u, v), y=\eta^{(2)}(u, v), z=\xi^{(2)}(u, v) \text {. }
$$

As before, the functions $\xi^{(2)}, \eta^{(2)}, \zeta^{(2)}$ continue to satisfy conditions (a), (b), (c), (e), and also

$$
F\left(\Sigma^{(2)}\right) \leqq F\left(\Sigma^{(1)}\right) \leqq F\left(\pi_{n}\right),
$$

where $\Sigma^{(2)}$ is the surface $x=\xi^{(2)}(u, v), \cdots,(u, v)$ on $K$. We repeat the process with $d+2 h / n$ in place of $d+1 / n$, obtaining the functions $\xi^{(3)}, \eta^{(3)}, \zeta^{(3)}$, and continue $n+1$ times, using the numbers $d+i h / n(i=3,4, \cdots, n)$ successively to obtain functions $\xi^{(n+1)}, \eta^{(n+1)}, \zeta^{(n+1)}$. We re-name these functions, calling them $\bar{\xi}_{n}, \bar{\eta}_{n}, \bar{z}_{n}$ respectively. They satisfy conditions (a), (b), (c), (e), and also

$$
F\left(\bar{\Sigma}_{n}\right) \leqq F\left(\pi_{n}\right),
$$

where $\bar{\Sigma}_{n}$ is the surface $x=\bar{\xi}_{n}(u, v)$, etc.

The set $\sum R_{i}$ is an open set, and its boundary, which consists of a finite number of analytic arcs, is of measure zero. Hence, neglecting a set of measure 0 , we have for $(u, v)$ in $K-\sum R_{i}$ the equality

$$
\bar{E}_{n}=E_{n}, \bar{G}_{n}=G_{n} \text {. }
$$

At each point of $\sum R_{i}$ the functions $\bar{\xi}_{n}$, etc., are defined by equations (3.19) or their analogues, so that by the use of elementary inequalities we find that

$$
\bar{E}_{n} \leqq k E_{n}, \bar{G}_{n} \leqq k G_{n},
$$

where $k$ is defined in (3.8). Hence, recalling inequality (3.18),

$$
\iint_{K}\left(\bar{E}_{n}+\bar{G}_{n}\right) d u d v \leqq k H .
$$

We readily see that the function $\bar{z}_{n}$ has a monotonic deficiency not greater than $h / n$. 
To the functions $\bar{\eta}_{n}$ of equations (3.3) we now apply a similar process. The points $(u, v)$ such that $\bar{\eta}_{n}(u, v)>d$ fall into a finite number of maximal connected sets; we reject those which have points in common with the circumference of $K$, and name the others $R_{1}, R_{2}, \cdots, R_{p}$. We treat the points $(u, v)$ for which $\bar{\eta}_{n}(u, v)<d$ similarly, obtaining sets $R_{p+1}, \cdots, R_{q}$. We now define

$$
\begin{aligned}
& \bar{\eta}^{(1)}=d,(u, v) \text { on } \sum R_{i}, \\
& \bar{\eta}^{(1)}=\bar{\eta}_{n},(u, v) \text { on } K-\sum R_{i} .
\end{aligned}
$$

The surface

(3.32) $\bar{\Sigma}^{(1)}: \quad x=\bar{\xi}_{n}(u, v), y=\bar{\eta}^{(1)}(u, v), z=\bar{z}_{n}(u, v),(u, v)$ on $K$, is easily seen to satisfy the inequality

$$
\iint_{K}\left(\bar{E}^{(1)}+\bar{G}^{(1)}\right) d u d v \leqq \iint_{K}\left(\bar{E}_{n}+\bar{G}_{n}\right) d u d v,
$$

where the functions $\bar{E}^{(1)}, \bar{G}^{(1)}$ correspond to $\bar{\Sigma}^{(1)}$ and $\bar{E}_{n}, \bar{G}_{n}$ to $\bar{\Sigma}_{n}$; for

$$
\bar{E}_{n}-\bar{E}^{(1)}
$$

has the value 0 on $K-\sum R_{i}$ and the value $\left(\partial \bar{\eta}_{n} / \partial u\right)^{2}$ on $\sum R_{i}$. Moreover, an argument similar to the above proves that

$$
F\left(\bar{\Sigma}^{(1)}\right) \leqq F\left(\bar{\Sigma}_{n}\right) \leqq F\left(\pi_{n}\right) ;
$$

we need only to permute $X, Y, Z$ cyclically and set $a=b=0$ in (3.23), recalling equations (2.10).

Applying the same process to the sets $R_{i}$ on which $\bar{\eta}^{(1)}>d+h / n$ or $\bar{\eta}^{(1)}<d+h / n$ gives $\bar{\eta}^{(2)}$; and continuing the process we obtain successively $\bar{\eta}^{(3)}, \cdots, \bar{\eta}^{(n+1)}$. The function $\bar{\eta}^{(n+1)}$ we re-name $\bar{y}_{n}$. Each alteration reduces (or leaves unchanged) the value of $\iint f d u d v$ and of $\iint(E+G) d u d v$, and leaves $\bar{z}_{n}(u, v)$ and $\bar{\xi}_{n}(u, v)$ unaltered.

Finally, we apply to the function $\bar{\xi}_{n}(u, v)$ the same process as we have just applied to $\bar{\eta}_{n}(u, v)$, arriving at a function $\bar{x}_{n}(u, v)$. We define the surface $\bar{S}_{n}$ by the equations

$$
\bar{S}_{n}: \quad x=\bar{x}_{n}(u, v), y=\bar{y}_{n}(u, v), z=\bar{z}_{n}(u, v),(u, v) \text { on } K .
$$

The following relations then hold:

$$
\begin{gathered}
F\left(\bar{S}_{n}\right) \leqq F\left(\pi_{n}\right), \\
\iint_{K}\left(\bar{E}_{n}+\bar{G}_{n}\right) d u d v \leqq k^{2} H .
\end{gathered}
$$


Moreover, the functions $x_{n}(u, v)$, etc., have monotonic deficiency not greater than $h / n$, and they satisfy conditions (a), (b), (c), and hence satisfy conditions (1.1). Since the functional values on the boundary have been left unaltered, it remains true that the points $A_{i}^{(n)}$ of $\Gamma_{n}$ correspond under (3.34) to the points $A_{i}^{*}$ of the circumference of $K$.

The hypotheses of Lemma 2 of S.S. are satisfied by the surfaces (3.34). Hence there exists a representation

$$
x=x(\theta), y=y(\theta), z=z(\theta), 0 \leqq \theta \leqq 2 \pi,
$$

of the curve $\Gamma$ and a subsequence $\left\{\bar{S}_{\alpha}\right\}$ of the $\left\{\bar{S}_{n}\right\}$, the subscript $\alpha$ ranging over a subset of the positive integers, such that $\dagger$

$$
\lim \bar{x}_{\alpha}(\theta)=x(\theta), \lim \bar{y}_{\alpha}(\theta)=y(\theta), \lim \bar{z}_{\alpha}(\theta)=z(\theta)
$$

uniformly in $\theta$.

Now by Lemma 1 of S.S. we can select a subsequence $\left\{\bar{S}_{\beta}\right\}$ of the sequence $\left\{\bar{S}_{\alpha}\right\}$ such that the functions $\bar{x}_{\beta}$ converge uniformly over the whole circle $K$ to a limit function $x(u, v)$. From the sequence $\left\{\bar{S}_{\beta}\right\}$ we can select a subsequence $\left\{\bar{S}_{\gamma}\right\}$ such that $\bar{y}_{\gamma}(u, v)$ converges uniformly on $K$ to a limit function $y(u, v)$. Finally, we can select a subsequence $\left\{\bar{S}_{\delta}\right\}$ of the sequence $\left\{\bar{S}_{\gamma}\right\}$ such that $\bar{z}_{\delta}$ converges uniformly on $K$ to a limit function $z(u, v)$. Moreover, by Lemma 1 of S.S. these limit functions are monotonic and the surface

$$
S: \quad x=x(u, v), y=y(u, v), z=z(u, v), u^{2}+v^{2} \leqq 1,
$$

satisfies conditions (1.1). From (3.37) we see that $S$ is bounded by $\Gamma$; hence

$$
F(S) \geqq i \text {. }
$$

By hypothesis the integral $F(S)$ is positive quasi-regular and positive definite; and we have just seen that the surfaces $\bar{S}_{n}, S$ all satisfy conditions (1.1), and by (3.36) their areas are uniformly bounded. Under these conditions it is known $\ddagger$ that $F(S)$ is lower semi-continuous, so that

$$
F(S) \leqq \lim \inf F\left(\bar{S}_{n}\right) .
$$

This, in conjunction with inequality (3.35) and equation (3.13), implies

$$
F(S) \leqq m \leqq i .
$$

Comparing inequalities (3.39) and (3.41) we find

$$
F(S)=i=m,
$$

and the theorem is proved.

$\dagger$ We here use $\bar{x}_{\alpha}(\theta)$ to denote $\bar{x}_{\alpha}(\cos \theta, \sin \theta)$, etc.

$\ddagger$ E. J. McShane, Integrals over surfaces in parametric form, Annals of Mathematics, vol. 34 (1933); in particular, Theorem III. 
4. Second existence theorem: non-definite integrals. If we restrict our attention to rectifiable curves $\Gamma$ and admit only comparison surfaces of type $\dagger$ $L_{2}$, the hypothesis that $F(S)$ is positive definite can be omitted, and we have

THEOREM 4.1. Let the integral

$$
F(S)=\iint f(X, Y, Z) d u d v
$$

be positive quasi-regular, and let $\Gamma$ be a rectifiable Jordan curve in xyz-space. Then in the class of all surfaces of type $L_{2}$ bounded $\ddagger$ by $\Gamma$ there exists a surface $S$ which minimizes $F(S)$.

We first show that for any constants $a, b, c$ the integral

$$
\iint_{K}(a X+b Y+c Z) d u d v
$$

has the same value for all surfaces

$$
x=x(u, v), y=y(u, v), z=z(u, v),(u, v) \text { on } K,
$$

bounded by $\Gamma$ and such that the functions $x(u, v)$, etc., satisfy conditions (1.1). (As before, $K$ is the unit circle.) Since the Dirichlet integrals of $x, y$ and $z$ are finite over $K$, the same is true of the harmonic functions $\xi, \eta, \zeta$ having the same boundary values as $x, y, z$ respectively. Reflecting these functions in the unit circumference yields harmonic functions $\bar{\xi}, \bar{\eta}, \bar{\zeta}$, defined outside of $K$, having finite Dirichlet integrals over the region $u^{2}+v^{2} \geqq 1$. Hence if we set $\bar{x}(u, v)=x(u, v)$ for $u^{2}+v^{2} \leqq 1$ and $\bar{x}(u, v)=\bar{\xi}(u, v)$ for $1<u^{2}+v^{2} \leqq 2$, and define $\bar{y}, \bar{z}$ analogously, the functions $\bar{x}, \bar{y}$, $\bar{z}$ satisfy conditions (1.1) over the whole circle $u^{2}+v^{2} \leqq 2$ and coincide with $x, y, z$ respectively for $u^{2}+v^{2} \leqq 1$.

We can now apply the proof of Lemma 4 of the last-cited paper, with the trivial change that the integrals $\int y z^{\prime} d t$, etc., are replaced by LebesgueStieltjes integrals $\int y d z$, etc.; we thus find that

$$
\iint_{K} X d u d v=\int y d z, \iint_{K} Y d u d v=\int z d x, \iint_{K} Z d u d v=\int x d y .
$$

The single integrals are taken around $\Gamma$ and are independent of the particular representation of $\Gamma$, and the invariance of the integral (4.2) follows at once.

\footnotetext{
$\dagger$ Defined at end of $\$ 1$.

$\ddagger$ Cf. footnote to Theorem 3.1.
} 
We treat separately the cases in which the $\varepsilon$-function $\mathcal{E}(X, Y, Z, \bar{X}, \bar{Y}, \bar{Z})$ is identically zero and that in which it is not identically zero. If it is identically zero, the integrand has the form

$$
f(X, Y, Z)=a X+b Y+c Z ;
$$

for

$$
\begin{aligned}
0= & (0,0,1, X, Y, Z)=f(X, Y, Z)-f(0,0,1) \\
& -X f_{X}(0,0,1)-Y f_{Y}(0,0,1)-(Z-1) f_{Z}(0,0,1) \\
= & f(X, Y, Z)-X f_{X}(0,0,1)-Y f_{Y}(0,0,1)-Z f_{Z}(0,0,1) .
\end{aligned}
$$

Hence the integral $F(S)$ has the same value for all surfaces under consideration, and if we choose any surface $S$ of type $L_{2}$ bounded by $\Gamma$ (the existence of such surfaces being obvious), it serves as a minimizing surface for $F(S)$.

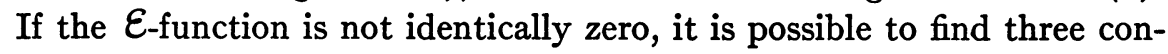
stants $a, b, c$ such that $\dagger$

$$
\phi(X, Y, Z) \equiv f(X, Y, Z)+a X+b Y+c Z>0
$$

for all $(X, Y, Z) \neq(0,0,0)$. For all surfaces of the type under consideration which are bounded by $\Gamma$, the integrals $F(S)$ and

$$
\Phi(S) \equiv \iint \phi(X, Y, Z) d u d v
$$

differ by a constant, hence a minimizing surface for $\Phi(S)$ is simultaneously a minimizing surface for $F(S)$. But because of inequality (4.3), Theorem 3.1 guarantees the existence of a minimizing surface of type $L_{2}$ for $\Phi(S)$, and our theorem is established.

† E. J. McShane, Remark concerning Mr. Graves' paper, etc., Monatshefte für Mathematik und Physik, vol. 39 (1932), p. 105. The proof applies without change to the present case.

PrINCETON UNIVERSITY, Princeton, N. J. 